\title{
Correction to: Extensive framework based on novel convolutional and variational autoencoder based on maximization of mutual information for anomaly detection
}

\author{
Qien $\mathrm{Yu}^{1} \cdot$ Muthu Subash Kavitha ${ }^{2,3} \cdot$ Takio Kurita $^{2}$
}

Published online: 2 August 2021

(C) Springer-Verlag London Ltd., part of Springer Nature 2021

Correction to: Neural Computing and Applications https://doi.org/10.1007/s00521-021-06017-3

In the original publication the name of one of the authors Publisher's Note Springer Nature remains neutral with regard to jurisdictional claims in published maps and institutional affiliations.

had been misspelled. The correct spelling is Muthu Subash Kavitha. The original article has been corrected.

The original article can be found online at https:// doi.org/10.1007/s00521-021-06017-3.

Takio Kurita

tkurita@hiroshima-u.ac.jp

Qien Yu

d182819@hiroshima-u.ac.jp

Muthu Subash Kavitha

Kavitha@hiroshima-u.ac.jp

1 Department of Information Engineering, Hiroshima University, Higashi-hiroshima, Hiroshima 739-8521, Japan

2 Graduate School of Advanced Science and Engineering, Hiroshima University, Higashi-hiroshima,

Hiroshima 739-8521, Japan

3 School of Information and DataSciences, Nagasaki University, Nagasaki 852-8521, Japan 